\title{
Harmonic regressor: Robust solution to least-squares problem
}

Proc IMechE Part l:

J Systems and Control Engineering

227(8) 662-668

(c) IMechE 2013

Reprints and permissions:

sagepub.co.uk/journalsPermissions.nav DOI: 10.1 I77/0959651813498873

pii.sagepub.com

(\$SAGE

\section{Alexander Stotsky}

\begin{abstract}
A new robust and computationally efficient solution to least-squares problem in the presence of round-off errors is proposed. The properties of a harmonic regressor are utilized for design of new combined algorithms of direct calculation of the parameter vector. In addition, an explicit transient bound for estimation error is derived for classical recursive least-squares algorithm using the Lyapunov function method. Different initialization techniques of the gain matrix are proposed as an extension of the recursive least-squares algorithm. All the results are illustrated by simulations.
\end{abstract}

\section{Keywords}

Recursive least-squares algorithm, harmonic regressor, strictly diagonally dominant matrix, high-order algorithms, recursive inversion

Date received: 19 March 2013; accepted: 2 July 2013

\section{Introduction}

Recursive least-squares (RLS) algorithms in which an estimate of the inverse of the information matrix is updated recursively are widely used in many applications such as adaptive control, signal processing, system identification and others. ${ }^{1}$ One of the main drawbacks of RLS algorithms is a round-off error accumulation problem, which in turn has a direct impact on the estimation performance and even on system stability. ${ }^{2-4}$ This problem might be solved via a frequent initialization of the gain matrix to the inverse of the information matrix. However, a computational burden associated with the inversion of the information matrix for largescale systems is the main obstacle for implementation of this technique.

The periodic nature of a wide class of machines results in oscillations in their signals. The model of oscillating signals can be presented in the vector form with a harmonic regressor, which consists of trigonometric functions (sines and cosines) at different frequencies. ${ }^{5}$ The information matrix of harmonic regressor, which is defined as the sum of rank 1 matrices calculated via multiplication of the regressor column vector by its transpose with a corresponding weighting sequence is a strictly diagonally dominant (SDD) matrix. ${ }^{6}$ Strict diagonal dominance of the information matrix is used in this article for improvement of robustness of RLS algorithms in the presence of round-off errors. The leastsquares problem is solved via direct calculation of the parameter vector with an SDD information matrix. This solution is preferable for large-scale systems (for signals with a large number of frequencies), where high accuracy of calculation of the coefficients is required. One of the algorithms for direct calculation of the coefficients of the trigonometric polynomial is reported in Stotsky. ${ }^{6}$ However, the convergence of algorithm described in that study ${ }^{6}$ might be slow. A new class of algorithms (highorder algorithms) for direct calculation of the parameters with improved convergence rate is proposed in this article.

Moreover, a new combination of the matrix inversion technique with a recursive algorithm of direct parameter calculation is also proposed in this article. This combination represents a two-stage algorithm, where an SDD information matrix is rapidly inverted with a certain (relatively low) accuracy at the first stage. Notice that the convergence rate of the matrix inversion algorithm is much higher than the convergence rate of the recursive algorithm of direct calculation of the parameters. Therefore, the matrix inversion algorithm is used in the initial steps. Further

Department of Signals and Systems, Chalmers University of Technology, Gothenburg, Sweden

\section{Corresponding author:}

Alexander Stotsky, Department of Signals and Systems, Chalmers University of Technology, Gothenburg SE-4I2 96, Sweden.

Email: alexander.stotsky@chalmers.se 
calculations of the inverse of an SDD information matrix give a minor contribution to the improvement of accuracy. A recursive algorithm for direct calculation of the parameter vector with improved convergence rate (high-order algorithm) is used in the second stage, and finally delivers the parameter vector with high accuracy. Simulations of new algorithms showed significant improvement of the estimation performance compared to well-known RLS algorithms in the presence of round-off errors.

Classical RLS algorithms are also studied in this article. Explicit transient bound for estimation error is derived using an assumption about positive definiteness of the gain update matrix. It is shown that the gain matrix, which is recursively updated, might be initialized differently to extend the capabilities of the recursive algorithm. It is also shown that RLS algorithm can be seen as a modification of the Kaczmarz projection algorithm, where the inverse of gain matrix is a positive definite and SDD matrix.

Main contributions of this article can be summarized as follows:

- A new class of high-order algorithms with improved convergence rate for direct calculation of the parameters in the least-squares problem;

- A new combined matrix inversion algorithm and recursive algorithm of direct parameter calculation;

- Explicit transient bound for estimation error in RLS algorithm;

- A new estimation algorithm in a form of RLS algorithm with an SDD gain matrix.

The article is organized as follows. The problem statement is presented in section "Problem statement." New properties of RLS algorithms are described in section "RLS algorithms." New recursive algorithms of direct calculation of the parameter vector are presented in section "Recursive algorithms of direct calculation of the parameter vector." Combined algorithms are presented in section "Combined recursive algorithms." The article ends with brief comparisons and conclusions in section "Other methods and conclusion."

\section{Problem statement}

Suppose that a measured oscillating signal $y_{k}$ can be presented in the following form

$$
y_{k}=\varphi_{k}^{T} \theta_{*}+\xi_{k}
$$

where $\varphi_{k}$ is the harmonic regressor and $\theta_{*}$ is the vector of constant unknown parameters, which are defined as follows

$$
\begin{aligned}
& \varphi_{k}^{T}=\left[1 \cos \left(q_{1} k\right) \sin \left(q_{1} k\right) \cos \left(q_{2} k\right) \sin \left(q_{2} k\right) \ldots\right. \\
& \left.\cos \left(q_{r} k\right) \sin \left(q_{r} k\right)\right] \\
& \theta_{*}^{T}=\left[\begin{array}{lllllll}
\theta_{0 *} & \theta_{1 *} & \theta_{2 *} & \theta_{3 *} & \theta_{4 *} & \ldots & \theta_{(2 r-1) *} \\
\theta_{(2 r) *}
\end{array}\right]
\end{aligned}
$$

where $k=1,2, \ldots$ is the step number; $q_{i} i=$ $1,2, \ldots, r$ are the frequencies; and $\xi_{k}$ is a zero mean white Gaussian noise.

The model of the signal (1) is presented in the following form

$$
\hat{y}_{k}=\varphi_{k}^{T} \theta_{k}
$$

with adjustable parameters

$$
\theta_{k}^{T}=\left[\begin{array}{lllllll}
\theta_{0 k} & \theta_{1 k} & \theta_{2 k} & \theta_{3 k} & \theta_{4 k} & \ldots & \theta_{(2 r-1) k} \\
\theta_{(2 r) k}
\end{array}\right]
$$

Minimization of the performance index

$$
E_{k}=\sum_{j=1}^{k} \beta_{j}\left(y_{j}-\hat{y}_{j}\right)^{2}
$$

where $\beta_{j}$ is a weighting sequence, results in the following least-squares solution

$$
\theta_{k}=\left[\sum_{j=1}^{k} \beta_{j} \varphi_{j} \varphi_{j}^{T}\right]^{-1} \sum_{j=1}^{k} \beta_{j} \varphi_{j} y_{j}
$$

Assigning weighting factor to one in step $k$ and to $\lambda_{0}$ in the previous steps, the equation (7) can be written as follows

$$
\begin{aligned}
& \theta_{k}=A_{k}^{-1} b_{k} \\
& A_{k}=\lambda_{0}\left[\sum_{j=1}^{k-1} \varphi_{j} \varphi_{j}^{T}\right]+\varphi_{k} \varphi_{k}^{T} \\
& b_{k}=\lambda_{0}\left[\sum_{j=1}^{k-1} \varphi_{j} y_{j}\right]+\varphi_{k} y_{k}
\end{aligned}
$$

where the matrix $A_{k}$ is information (Hessian) matrix, and $0<\lambda_{0}<1$ is a forgetting factor, $k=2,3 \ldots$.

In order to calculate the parameter vector $\theta_{k}$, this information matrix should be inverted in each step $k$. This is a computationally expensive procedure, if the size of the parameter vector is large. This matrix is an SDD matrix for a sufficiently large $k$ and forgetting factor, which is close to one, ${ }^{7}$ that facilitates matrix inversion and allows direct calculation of the parameter vector.

\section{RLS algorithms}

\section{Algorithm description and transient bound on estimation error}

RLS solution of equation (8) with $\Gamma_{k}=A_{k}^{-1}$ can be written as follows ${ }^{1}$

$$
\begin{aligned}
& \theta_{k}=\theta_{k-1}+\frac{\Gamma_{k-1} \varphi_{k}}{\lambda_{0}+\varphi_{k}^{T} \Gamma_{k-1} \varphi_{k}}\left(y_{k}-\theta_{k-1}^{T} \varphi_{k}\right) \\
& \Gamma_{k}=\frac{1}{\lambda_{0}}\left[\Gamma_{k-1}-\frac{\Gamma_{k-1} \varphi_{k} \varphi_{k}^{T} \Gamma_{k-1}}{\left(\lambda_{0}+\varphi_{k}^{T} \Gamma_{k-1} \varphi_{k}\right)}\right]
\end{aligned}
$$

The properties of the systems (1), (4), (11) and (12) are summarized in the following Lemma. 
Lemma. Consider the systems (1) and (4) with $\xi_{k}=0$ and recursive estimation algorithms (11) and (12). Assume that the following inequalities for the inverse of the gain matrix are valid

$$
0<\alpha_{1} I \leqslant \Gamma_{k}^{-1} \leqslant \alpha_{2} I
$$

where $\alpha_{1}$ and $\alpha_{2}$ are positive constants and $I$ is the identity matrix. Then the following bound for estimation error $\tilde{\theta}_{k}=\theta_{k}-\theta_{*}$ is valid

$$
\left\|\tilde{\theta}_{k}\right\| \leqslant \sqrt{\frac{\lambda_{0}^{k} V_{0}}{\alpha_{1}}}
$$

where $V_{0}=\tilde{\theta}_{0}^{T} \Gamma_{0}^{-1} \tilde{\theta}_{0}$ and $\tilde{\theta}_{0}=\theta_{0}-\theta_{*}$

Proof. The following relations are valid for algorithms (11) and (12)

$$
\begin{aligned}
& \Gamma_{k}^{-1}=\lambda_{0} \Gamma_{k-1}^{-1}+\varphi_{k} \varphi_{k}^{T} \\
& \tilde{\theta}_{k}-\tilde{\theta}_{k-1}=-\frac{\Gamma_{k-1} \varphi_{k} \varphi_{k}^{T}}{\lambda_{0}+\varphi_{k}^{T} \Gamma_{k-1} \varphi_{k}} \tilde{\theta}_{k-1} \\
& \varphi_{k} \varphi_{k}^{T} \tilde{\theta}_{k}=\frac{\lambda_{0} \varphi_{k} \varphi_{k}^{T}}{\lambda_{0}+\varphi_{k}^{T} \Gamma_{k-1} \varphi_{k}} \tilde{\theta}_{k-1}
\end{aligned}
$$

where equation (15) follows from relation (9), equation (16) follows from relation (11) and finally equation (17) follows from relation (16) when multiplying relation (16) by $\varphi_{k} \varphi_{k}^{T}$.

The performance of RLS algorithm is evaluated using Lyapunov function candidate $V_{k}=\tilde{\theta}_{k}^{T} \Gamma_{k}^{-1} \tilde{\theta}_{k}$, which can be written as $V_{k}=\tilde{\theta}_{k}^{T}\left(\lambda_{0} \Gamma_{k-1}^{-1}+\varphi_{k} \varphi_{k}^{T}\right) \tilde{\theta}_{k}$. Taking into account equations (16) and (17), the first difference is evaluated as follows

$$
\begin{aligned}
V_{k}-V_{k-1} & =\lambda_{0}\left[\tilde{\theta}_{k}^{T} \Gamma_{k-1}^{-1} \tilde{\theta}_{k}-\tilde{\theta}_{k-1}^{T} \Gamma_{k-1}^{-1} \tilde{\theta}_{k-1}\right] \\
& +\tilde{\theta}_{k}^{T} \varphi_{k} \varphi_{k}^{T} \tilde{\theta}_{k}+\left(\lambda_{0}-1\right) \tilde{\theta}_{k-1}^{T} \Gamma_{k-1}^{-1} \tilde{\theta}_{k-1} \\
& =\lambda_{0}\left(\tilde{\theta}_{k}-\tilde{\theta}_{k-1}\right)^{T} \Gamma_{k-1}^{-1}\left(\tilde{\theta}_{k}+\tilde{\theta}_{k-1}\right) \\
& +\lambda_{0} \tilde{\theta}_{k}^{T} \frac{\varphi_{k} \varphi_{k}^{T}}{\lambda_{0}+\varphi_{k}^{T} \Gamma_{k-1} \varphi_{k}} \tilde{\theta}_{k-1} \\
& +\left(\lambda_{0}-1\right) \tilde{\theta}_{k-1}^{T} \Gamma_{k-1}^{-1} \tilde{\theta}_{k-1} \\
& =-\lambda_{0} \tilde{\theta}_{k-1}^{T} \frac{\varphi_{k} \varphi_{k}^{T}}{\lambda_{0}+\varphi_{k}^{T} \Gamma_{k-1} \varphi_{k}} \tilde{\theta}_{k-1} \\
& +\left(\lambda_{0}-1\right) \tilde{\theta}_{k-1}^{T} \Gamma_{k-1}^{-1} \tilde{\theta}_{k-1}
\end{aligned}
$$

and $V_{k} \leqslant \lambda_{0} V_{k-1}$. Hence the bound (14) is true.

\section{Initialization of the gain matrix $\Gamma_{k}$ : two algorithms in one form}

For convergence of the recursive algorithms (11) and (12), inequalities (13) should be satisfied. The gain matrix might be initialized in different ways in order to satisfy inequalities (13). Moreover, the diagonal dominance property of matrix $A_{k}$ due to harmonic regressor facilitates the choice of initial gain matrix and extends the capabilities of the recursive algorithms (11) and (12).

Two initialization techniques can be used in algorithms (11) and (12), where the matrix $\Gamma_{k}$ plays different roles. Initialization of the gain matrix $\Gamma_{k}$ to the inverse of the information matrix $A_{k}$ specifies the algorithm (12) as a recursive estimate of the inverse of the information matrix. This case corresponds to classical RLS algorithm. Initialization of the matrix $\Gamma_{k}$ to a diagonal matrix with positive diagonal elements allows to treat this matrix as a gain matrix, and the properties of the algorithms (11) and (12) become similar to the properties of the Kaczmarz algorithm. ${ }^{7,8}$ Classical RLS algorithms (11) and (12) are robust with respect to deviations of the gain matrix $\Gamma_{k}$ from the inverse of the information matrix $A_{k}$, provided that inequalities (13) are valid.

Comparison of these two algorithms is presented in Figure 1, which shows time evolution of three estimated parameters defined in equation (5). Figure 1 shows that classical RLS algorithm provides better estimation performance compared to recursive estimation algorithm with an SDD gain matrix. The latter, however, is more robust against error accumulation problem, and its properties are similar to the properties of the Kaczmarz algorithm.,

\section{Recursive algorithms of direct calculation of the parameter vector}

\section{Second-order algorithm}

Least-squares problem is a problem of calculation of the parameter vector $\theta_{k}$ with high accuracy. The parameter vector $\theta_{k}$ in equation (8) can be recursively estimated as follows

$$
\begin{gathered}
\vartheta_{i}=\vartheta_{i-1}-2 D_{k}^{-1} A_{k} \vartheta_{i-1}+2 D_{k}^{-1} b_{k}+ \\
\left(D_{k}^{-1} A_{k}\right)^{2} \vartheta_{i-1}-D_{k}^{-1} A_{k} D_{k}^{-1} b_{k}
\end{gathered}
$$

where $\vartheta_{i}$ is an estimate of $\theta_{k} ; D_{k}$ is a diagonal matrix that contains diagonal elements of $A_{k}$, which is defined in equation (9); and $b_{k}$ is defined in equation (10). Equations (8) and (18) can be combined as follows

$$
\tilde{\vartheta}_{i}=\left(I-D_{k}^{-1} A_{k}\right)^{2} \tilde{\vartheta}_{i-1}
$$

where $\tilde{\vartheta}_{i}=\vartheta_{i}-\theta_{k}$ is estimation error.

The following inequality is valid $\left\|I-D_{k}^{-1} A_{k}\right\| \leqslant \kappa<1$ due to the diagonal dominance of the matrix $A_{k}, 6,9$ where the norm $\|\cdot\|$ is defined as the maximum row sum matrix norm. This inequality guarantees the stability of the error model (19).

Notice that two errors are defined as estimation errors in this article. The first one is the error between estimated parameter vector $\theta_{k}$ and true parameter vector $\theta_{*}$. This error is associated with estimation performance of RLS algorithm, see section "RLS algorithms." The second estimation error $\vartheta_{i}-\theta_{k}$ is 


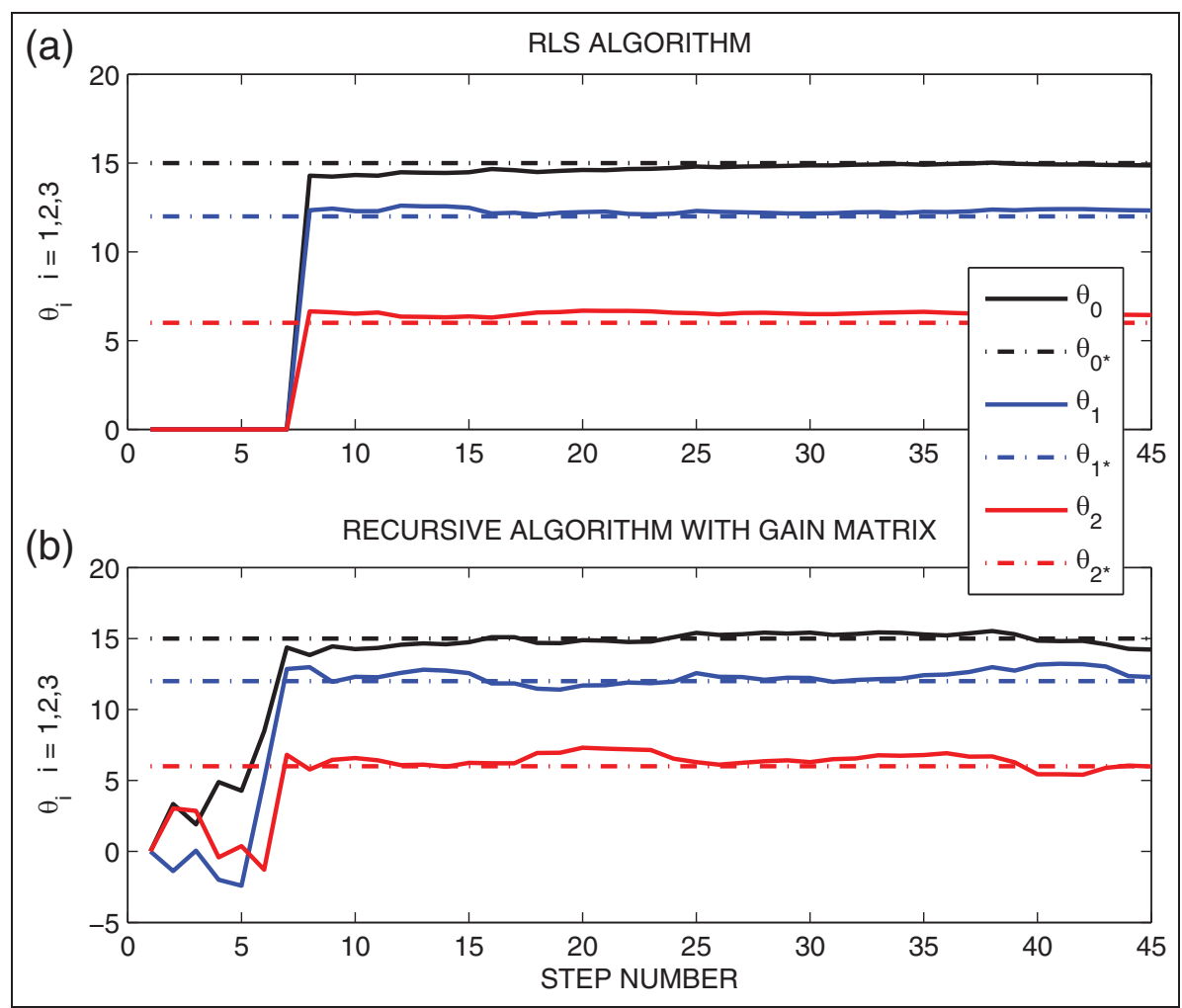

Figure I. Comparison of (a) RLS algorithm and (b) recursive algorithm with an SDD gain matrix applied for estimation of the parameters of the noise-contaminated signal that contains three frequencies. Estimated parameters, defined in equation (5) are plotted with solid black, blue and red lines, whereas true parameters are plotted with dash-dot lines of the same colors.

associated with numerical calculation of the parameter vector $\theta_{k}$ defined in equation (8).

The following inequality is valid for estimation error $\tilde{\vartheta}_{i}$

$$
\left\|\tilde{\vartheta}_{i}\right\| \leqslant \kappa^{2 i}\left\|\tilde{\vartheta}_{0}\right\|
$$

and $\vartheta_{i}$ rapidly converges to $\theta_{k}$.

Notice that algorithm (18) is named in this article as a second-order algorithm due to the square of the error matrix $\left(I-D_{k}^{-1} A_{k}\right)^{2}$ in the error model (19). This algorithm has improved converge rate compared to the first-order algorithm, described in Stotsky. ${ }^{6}$ Notice that the second-order algorithm requires more computational efforts than a first-order algorithm.

The algorithm (18) is stopped if

$$
\left\|A_{k} \vartheta_{i}-b_{k}\right\|<\epsilon
$$

where $\epsilon$ is a sufficiently small positive number that controls the accuracy of estimation.

Notice that the diagonal matrix $D_{k}^{-1}$ in algorithm (18) plays a role of a priori estimate of the inverse of $A_{k}$. Obviously, the convergence rate of algorithm (18) can be improved via improvement of the estimate of the inverse of $A_{k}$. Such algorithms are described in section "Combined recursive algorithms."

\section{High-order algorithms}

Algorithms of higher order can be worked out from the following error model

$$
\tilde{\vartheta}_{i}=\left(I-G A_{k}\right)^{n} \tilde{\vartheta}_{i-1}
$$

where $n=1,2,3, \ldots$ and $G$ is an estimate of the matrix $A_{k}^{-1}$, such that $\left\|I-G A_{k}\right\| \leqslant \kappa<1$, for example, $G=D_{k}^{-1}$. Algorithms of the order $n$ can be written as follows

$$
\begin{aligned}
\vartheta_{i} & =\vartheta_{i-1}+\left\{\sum_{d=0}^{n-1} \frac{n !}{d !(n-d) !}(-1)^{n-d}\left(G A_{k}\right)^{n-d}\right\} \vartheta_{i-1} \\
& +\sum_{d=0}^{n-1} \frac{n !}{d !(n-d) !}(-1)^{n-d-1}\left(G A_{k}\right)^{n-d-1} G b_{k}
\end{aligned}
$$

and are summarized in Table 1.

Notice that $n$ iterations of the first-order algorithm are performed in each step in the $n$ th-order algorithm. High-order algorithms might be useful in the estimation problems where a pre-specified accuracy should be achieved in a certain number of steps. The order of the algorithm can be chosen in this case using the error model (22).

\section{Combined recursive algorithms}

This algorithm starts with a recursive estimation of the inverse of matrix $A_{k}$ with the following initialization

$$
\begin{aligned}
& F_{0}=I-G_{0} A_{k} \\
& G_{0}=D_{k}^{-1}
\end{aligned}
$$


Table I. A family of high-order algorithms.

\begin{tabular}{ll}
\hline Order & High-order algorithms \\
\hline 1 & $\vartheta_{i}=\vartheta_{i-1}-G\left(A_{k} \vartheta_{i-1}-b_{k}\right)$ \\
2 & $\vartheta_{i}=\vartheta_{i-1}-2 G A_{k} \vartheta_{i-1}+2 G b_{k}+\left(G A_{k}\right)^{2} \vartheta_{i-1}-G A_{k} G b_{k}$ \\
3 & $\vartheta_{i}=\vartheta_{i-1}-\left(G A_{k}\right)^{3} \vartheta_{i-1}+3\left(G A_{k}\right)^{2} \vartheta_{i-1}-3 G A_{k} \vartheta_{i-1}+\left(G A_{k}\right)^{2} G b_{k}-3 G A_{k} G b_{k}+3 G b_{k}$ \\
& $\cdots$ \\
$n$ & $\vartheta_{i}=\vartheta_{i-1}+\left\{\sum_{d=0}^{n-1} \frac{n !(n-d) !}{}(-1)^{n-d}\left(G A_{k}\right)^{n-d}\right\} \vartheta_{i-1}+\sum_{d=0}^{n-1} \frac{n !}{d !(n-d) !}(-I)^{n-d-1}\left(G A_{k}\right)^{n-d-1} G b_{k}$
\end{tabular}

$$
\left\|F_{0}\right\| \leqslant \kappa<1
$$

where $D_{k}$ is a diagonal matrix that contains diagonal elements of $A_{k}$. Matrix $G_{i}$ is an estimate of the inverse of $A_{k}$, which is updated if the norm of the inversion error $I-G_{i-1} A_{k}$ is larger than a pre-specified number $\delta>0$ as follows

$$
\begin{aligned}
& \text { If }\left\|I-G_{i-1} A_{k}\right\| \geqslant \delta \\
& G_{i}=G_{i-1}+F_{i-1} G_{i-1} \\
& F_{i}=I-G_{i} A_{k}
\end{aligned}
$$

else

$$
G_{i}=G_{i-1}
$$

The matrix $G_{i}$ is used as an input to the update law for the parameter vector $\vartheta_{i}$

$$
\begin{gathered}
\vartheta_{i}=\vartheta_{i-1}-2 G_{i} A_{k} \vartheta_{i-1}+2 G_{i} b_{k}+ \\
\left(G_{i} A_{k}\right)^{2} \vartheta_{i-1}-G_{i} A_{k} G_{i} b_{k}
\end{gathered}
$$

The error model for this algorithm (without condition (27)) can be written as

$$
\tilde{\vartheta}_{i}=F_{0}^{2^{i+1}} \tilde{\vartheta}_{i-1}
$$

which guarantees very fast convergence of $\vartheta_{i}$ to $\theta_{k}$.

If algorithms (27)-(29) are executed for several steps $i=1, \ldots, w$ for a sufficiently large $\delta$, then the model for estimation error for $i=w+1, w+2, \ldots$ can be written as follows

$$
\tilde{\vartheta}_{i}=F_{0}^{2^{w+1}} \tilde{\vartheta}_{i-1}
$$

where $\left\|F_{0}^{2^{w+1}}\right\| \leqslant \kappa_{1}<\kappa<1$ and a positive constant $\kappa_{1}$ define the convergence rate of the model (32).

The algorithm is stopped, if $\left\|A_{k} \vartheta_{i}-b_{k}\right\|<\varepsilon$, where $\varepsilon$ is a sufficiently small positive number. Notice that a recursive matrix inversion is a computationally expensive procedure, and therefore, the inverse of the information matrix might be calculated with low accuracy. Estimation of the inverse matrix is stopped if $\left\|I-G_{i} A_{k}\right\|<\delta$, where the norm $\|\cdot\|$ is the maximum row sum matrix norm, and the accuracy of the parameter calculation is much higher than an accuracy of the matrix inversion $\delta \ggg \varepsilon$.
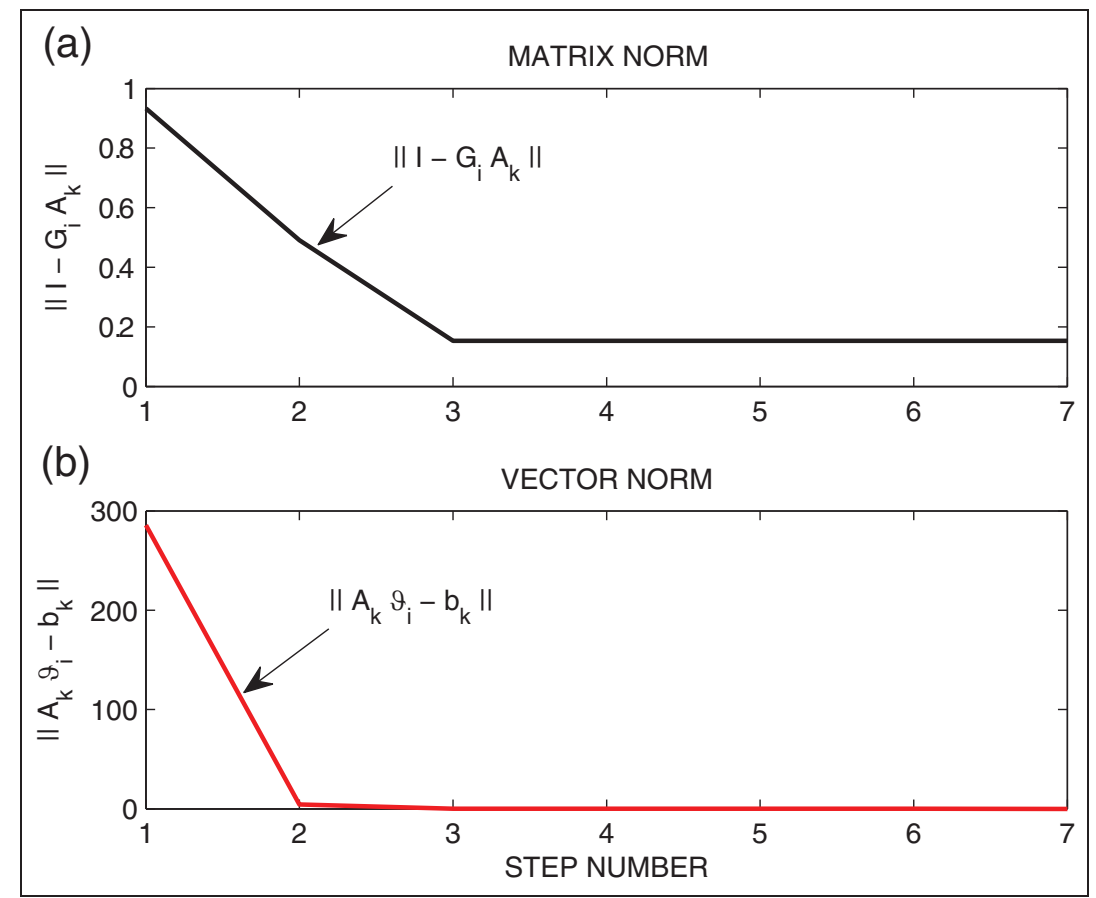

Figure 2. Time evolution of the (a) matrix norm $\left\|I-G_{i} A_{k}\right\|$ and (b) vector norm $\left\|A_{k} \vartheta_{i}-b_{k}\right\|$ for algorithms (24)-(30) with $\delta=0.155$ and $\epsilon=710^{-7}$. 


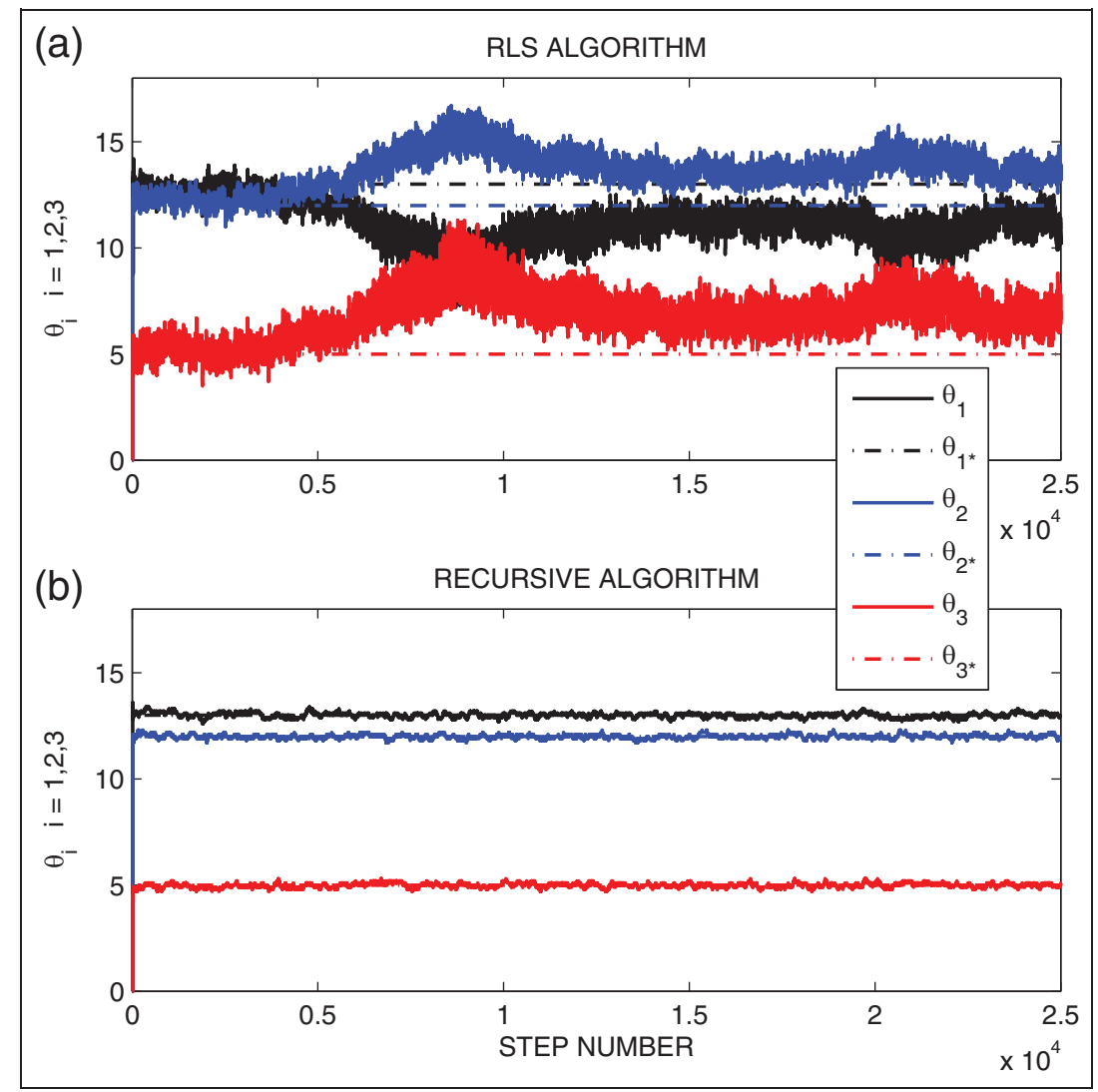

Figure 3. Comparison of (a) RLS algorithms (II) and (I2) and (b) combined recursive algorithms (24)-(30) for direct calculation of the parameters of the noise-contaminated signal that contains three frequencies. Estimated parameters are plotted with solid black, blue and red lines, whereas true parameters are plotted with dash-dot lines of the same colors. All the variables in both algorithms are rounded to the first (leading) digit.

The performance of algorithms (24)-(30) is illustrated in Figure 2, where a time evolution of the norms $\left\|I-G_{i} A_{k}\right\|$ and $\left\|A_{k} \vartheta_{i}-b_{k}\right\|$, which represent the matrix inversion and parameter estimation errors, is presented. The matrix norm $\left\|I-G_{i} A_{k}\right\|$ is sufficiently reduced in three steps, and therefore, the matrix $G_{i}$ gets sufficiently close to the inverse of information matrix $A_{k}$. Simultaneously, the vector norm $\left\|A_{k} \vartheta_{i}-b_{k}\right\|$ is essentially reduced in three steps. The accuracy which is required for a high-performance parameter estimation is achieved using algorithm (30) in the subsequent steps, see the second subplot of Figure 2.

Finally, a comparison of RLS algorithms (11) and (12) and combined recursive algorithms (24)-(30) for direct calculation of the parameters of the noisecontaminated signal that contains three frequencies is presented in Figure 3. The components of parameter vector (11) and the elements of gain matrix (12) of classical RLS algorithm are rounded to the first digit. All variables in combined recursive algorithms (24)-(30) are also rounded to the first digit, whereas the information matrix $A_{k}$, defined in equation (9) is calculated with higher accuracy. Rounding to the first digit is seldom met in practice, but it emphasizes the error accumulation problem in classical RLS algorithm. Figure 3 shows that the algorithms (24)-(30) provide better estimation performance. This is maximum possible performance improvement when using the approach proposed in this article.

\section{Other methods and conclusion}

Two groups of methods might be used to solve linear algebraic equation $A_{k} \theta_{k}=b_{k}$ : exact methods and recursive methods. Gaussian elimination method is the best known exact method for calculation of the parameter vector. ${ }^{10,11}$ Unfortunately, this method does not give an exact solution of the algebraic equations due to roundoff errors, which are always present in a finite precision implementation environment, and becomes computationally expensive for signals with a large number of frequencies.

Recursive methods for solution of linear algebraic equations, described in this article, that are based on strict diagonal dominance of the information matrix are preferable for large-scale systems as fast, robust and computationally efficient algorithms with controllable accuracy, which are suitable for real-time implementation.

This article shows that the properties of the harmonic regressor might be successfully used for 
robustification of RLS algorithms. Moreover, explicit transient bound for estimation error was derived in this article using Lyapunov function method for classical RLS algorithms. It was also shown that RLS algorithms can be used for the parameter estimation without initialization of the gain matrix to the inverse of the information matrix. This in turn allows improvement of the computational efficiency and shows robustness of RLS algorithms.

\section{Declaration of conflicting interests}

The author declares that there is no conflict of interest.

\section{Funding}

This research received no specific grant from any funding agency in the public, commercial or not-for-profit sectors.

\section{References}

1. Ljung L. System identification: theory for the user. Upper Saddle River, NJ: Prentice Hall, 1999.

2. Ljung $\mathrm{S}$ and Ljung L. Error propagation properties of recursive least-squares adaptation algorithms. Automatica 1985; 21(2): 157-167.
3. Verhaegen $\mathrm{M}$ and Van Dooren P. Numerical aspects of different kalman filter implementations. IEEE T Automat Contr 1986; 31(10): 907-917.

4. Liavas A and Regalia P. On the numerical stability and accuracy of the conventional recursive least squares algorithm. IEEE T Signal Proces 1999; 47(1): 88-96.

5. Bayard D. A general theory of linear time-invariant adaptive feedforward systems with harmonic regressors. IEEE T Automat Contr 2000; 45(11): 1983-1996.

6. Stotsky A. Recursive trigonometric interpolation algorithms. Proc IMechE, Part I: J Systems and Control Engineering 2010; 224(1): 65-77.

7. Stotsky A. A new frequency domain system identification method. Proc IMechE, Part I: J Systems and Control Engineering 2012; 226: 111-124.

8. Avedyan E. Modified Kaczmarz algorithms for estimating the parameters of linear plants. Automat Rem Contr 1978; 39(5): 674-680.

9. Horn R and Johnson C. Matrix analysis. Cambridge: Cambridge University Press, 1985.

10. Atkinson K. An introduction to numerical analysis. 2nd ed. New York: John Wiley \& Sons, 1989.

11. Ye Q. Computing singular values of diagonally dominant matrices to high relative accuracy. Math Comput 2008; 77(264): 2195-2230. 